\title{
COMPARISON BETWEEN SPARC E-METER MEASUREMENTS AND SIMULATIONS*
}

\author{
C. Ronsivalle, L. Giannessi, M. Quattromini, ENEA C.R. Frascati, Frascati(Roma), Italy \\ A. Bacci, A.R. Rossi,L. Serafini, INFN-Milano, Milano, Italy \\ M. Boscolo, E. Chiadroni, M. Ferrario, D. Filippetto, V. Fusco, G. Gatti, M. Migliorati, A. Mostacci, \\ C. Vaccarezza, C. Vicario, INFN/LNF, Frascati(Roma), Italy \\ A. Cianchi, INFN-Roma II, Roma, Italy \\ M. Petrarca, Università di Roma I La Sapienza, Roma, Italy
}

\section{Abstract}

For the SPARC photoinjector commissioning the emittance compensation process has been studied experimentally under different beam conditions (variation of charge, spot size, beam shape....) by a novel device called "emittance-meter", consisting in a movable emittance measurement system based on the 1D pepper pot method scanning a region $1.2 \mathrm{~m}$ long downstream the RF-gun. The results of a detailed comparison between the measurements and beam dynamics simulations performed by the codes (PARMELA,HOMDYN,TREDI) employed for SPARC design are presented and discussed here.

\section{STRATEGY FOR COMPARISON}

During the first stage of the SPARC photoinjector commissioning aimed to characterize the beam at the exit of the RF gun, the beam size, the emittance and the energy-spread at different $\mathrm{z}$ positions from the cathode have been measured by using the so-called movable emittance-meter [1]. This novel device allowed to perform very detailed tests of beam dynamics numerical codes.

All the simulations have been performed by PARMELA [2], which has been extensively used in the SPARC design. Crosschecks with other codes also employed for SPARC beam dynamics studies, TREDI [3] and HOMDYN [4], have been done as well.

\section{Fitting Procedure}

The aim of the measurements-simulation comparison was to verify the consistency of the experimental data with a numerical model describing a beam equivalent to the real one in conditions near to the machine operation.

The emittance is a function of the different parameters listed in table 1 with their uncertainties. These uncertainties represent the variation range of the parameters used in the numerical model to fit the emittance measurements.

The first step of the fitting procedure has been the matching of the measured envelope by adjusting the two parameters that mainly affect it, i.e. the initial spot size and the magnetic field strength, and keeping the other parameters fixed to their nominal value. Afterwards a fine adjustment has been done by tuning the other parameters.

*Work supported by the EU Commission in the sixth framework program, Contract No. 011935 EUROFEL.
Table 1: Variation Range Of Parameters

\begin{tabular}{|l|l|}
\hline Parameter & Variation range \\
\hline Energy & Measured $\pm 5 \%$ \\
\hline Charge & Measured $\pm 10 \%$ \\
\hline Spot size & $\begin{array}{l}\text { Retrieved from the virtual } \\
\text { cathode image } \pm 10 \%\end{array}$ \\
\hline Solenoid current & Measured $\pm 0.5 \%$ \\
\hline Rf phase & Measured $\pm 2^{\circ}$ \\
\hline
\end{tabular}

\section{Beam Model}

In PARMELA simulations the input beam longitudinal distribution has been directly retrieved by the measured temporal distribution. The two different techniques used to reconstruct the time profile based respectively on a cross-correlator and a spectrometer give a resolution of $0.5 \mathrm{psec}$. This value has been assumed as to the precision of the pulse length determination. The input transverse distribution has been retrieved from the so-called "virtual cathode" image obtained splitting the laser beam before it enters the vacuum system. It is generated cutting the edges of a transverse Gaussian profile in order to obtain an almost round and homogeneous intensity distribution and can be approximated by two types of 2D models: a truncated gaussian or a stack of uniform disks. The use of these 2D models in PARMELA ( $\mathrm{Nr}=$ number of radial meshes $=20, N z=$ number of longitudinal meshes $=200$, a radial mesh size automatically adjusted by the code, a variable longitudinal mesh size between 1 and $0.5 \mathrm{~cm}$, $\mathrm{Np}=$ number of particles $=20 \mathrm{~K}$ ) gives the possibility to get a fast tuning of fitting parameters. Checks by more time consuming $3 \mathrm{D}$ computations $(\mathrm{Np}=100 \mathrm{~K})$ showed that the above approximations are very satisfying in most of the cases. The same approach has been adopted also in TREDI simulations.

\section{Experimental Data Representation}

A code dedicated to the data analysis reconstructs in a very accurate way the emittance from acquired pepper-pot images and estimates the relative uncertainty as the experimental standard deviation of the mean [5]. This uncertainty multiplied by a factor corresponding to a gaussian confidence interval of $95 \%$ determines the error bars associated to the experimental data and shown in the plots. 


\section{COMPARISON RESULTS}

\section{Measured Emittance for Different Pulse Shapes}

The dynamic behaviour induced by different pulse shapes during the emittance compensation process was extensively investigated. The emittance-envelope plot of fig. 1 shows an optimum agreement between measurements and PARMELA simulations for a gaussian pulse with a FWHM of $10 \mathrm{psec}$ and the parameters reported in the second column of table 2 .
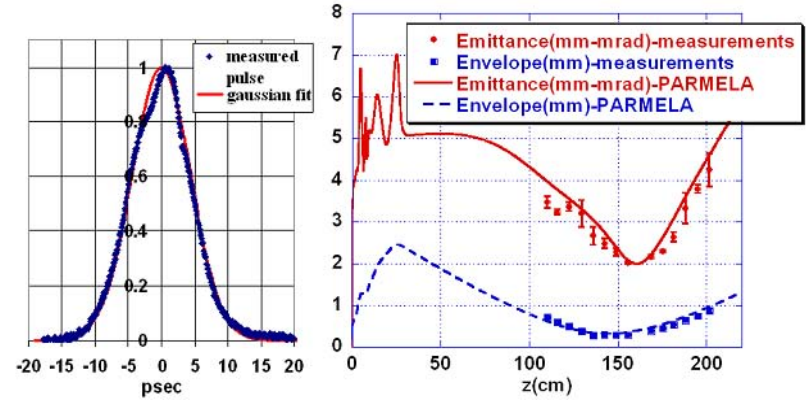

Figure 1: Measured pulse and corresponding envelopeemittance plot: measurements and PARMELA results.

A direct comparison of different pulse shapes is shown in fig. 2: both pulses have a FWHM of 8.7 psec (the other parameters are in the third column of table 2) and the comparison puts in evidence that the minimum emittance value is reduced from 2 to $1.5 \mathrm{~mm}$-mrad by using a flattop shape instead of a gaussian pulse, accordingly with PARMELA simulations.
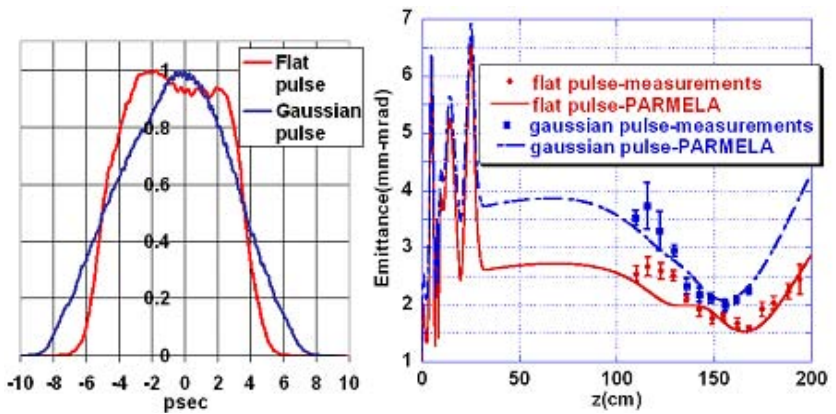

Figure 2: Measured pulses and corresponding emittance plots with measurements and PARMELA results.

The best brightness was achieved by the flat-top pulse (FWHM=8.9 psec) shown in fig.3 together with the PARMELA simulation based on the parameters of the fourth column of table 2 .
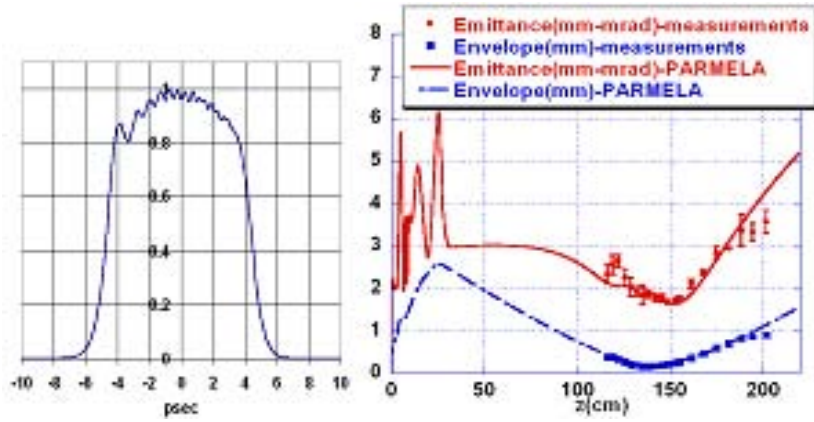

Figure 3: Measured pulse and corresponding emittance envelope plot with measurements and PARMELA results.
A crosscheck of PARMELA fit with TREDI code was done in this case (fig. 4) showing a satisfying agreement between measurements and numerical predictions within the measures uncertainties and the intrinsic differences between the two codes.

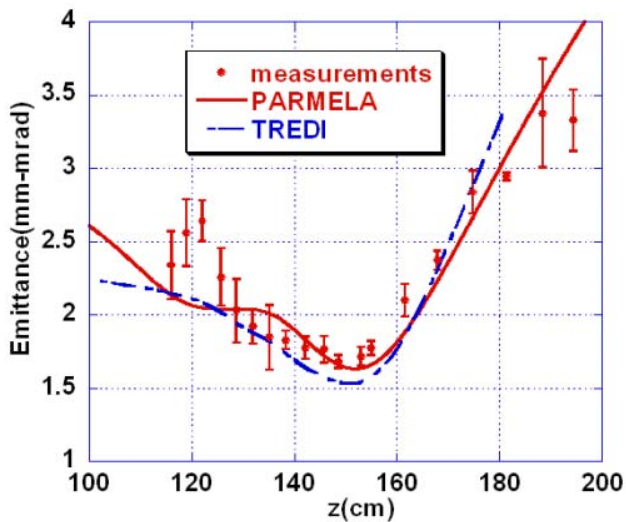

Figure 4: Emittance plot with PARMELA-TREDI vs measurements for the case of fig. 4 .

Table 2: Measured Parameters

\begin{tabular}{|l|l|l|l|}
\hline Parameter & $\begin{array}{l}\text { Case of } \\
\text { fig.1 }\end{array}$ & $\begin{array}{l}\text { Case of } \\
\text { fig.2 }\end{array}$ & $\begin{array}{l}\text { Case of } \\
\text { fig.3 }\end{array}$ \\
\hline Energy & $5.65 \mathrm{MeV}$ & $5.4 \mathrm{MeV}$ & $5.65 \mathrm{MeV}$ \\
\hline Charge & $1 \mathrm{nC}$ & $0.74 \mathrm{nC}$ & $0.825 \mathrm{nC}$ \\
\hline Spot size, $<\sigma>$ & $450 \mu \mathrm{m}$ & $310 \mu \mathrm{m}$ & $360 \mu \mathrm{m}$ \\
\hline Rf phase, $\varphi-\varphi_{\max } *$ & $-5^{\circ}$ & $-8^{\circ}$ & $-8^{\circ}$ \\
\hline Solenoid current & $209 \mathrm{~A}$ & $199 \mathrm{~A}$ & $209 \mathrm{~A}$ \\
\hline
\end{tabular}

$*(\varphi m a x=$ phase corresponding to the maximum energy gain $)$

All the above flat-top pulses have a rise/fall time around $2.6 \mathrm{psec}$. A reduction of the rise time to $\sim 1.5 \mathrm{psec}$ was obtained by shorting the pulse length to $\sim 5$ psec.

As it is shown in figure 5 such short rise time pulse allowed to measure for the first time the so-called "double emittance minimum" observed up to now only in numerical simulations. The oscillation was put in evidence by moving the phase of 12 degrees behind the phase of maximum energy gain (see table 3 ) in order to increase the beam energy spread accordingly with the chromatic nature of the effect [6].
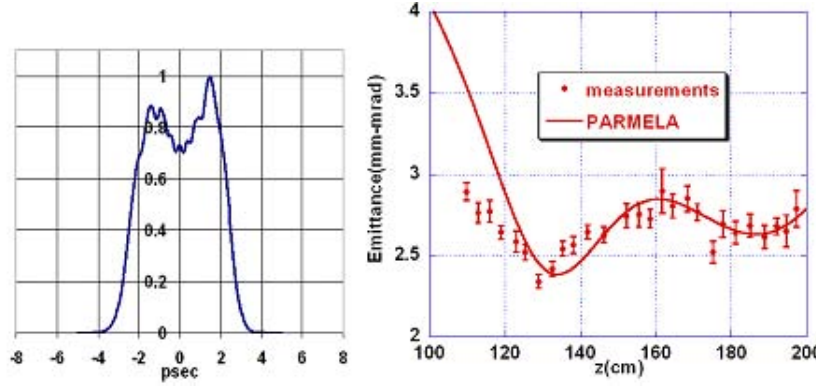

Figure 5: Measured pulse and corresponding emittance plot with measurements and PARMELA results. 
Table 3: Measured Parameters

\begin{tabular}{|l|l|}
\hline Parameter & Value \\
\hline Energy & $5.5 \mathrm{MeV}$ \\
\hline Charge & $0.5 \mathrm{nC}$ \\
\hline Spot size, $<\sigma>$ & $450 \mu \mathrm{m}$ \\
\hline Rf phase, $\varphi-\varphi_{\max }$ & $+12^{\circ}$ \\
\hline Solenoid current & $198 \mathrm{~A}$ \\
\hline
\end{tabular}

\section{Energy Spread Vs Z Measurements}

Figure 6 shows the evolution of the energy spread measured by cutting the beam by one slit at different $\mathrm{z}$ positions for two different values of beam charge.

The results have been compared with PARMELA simulations based on the measured parameters of table 4 .

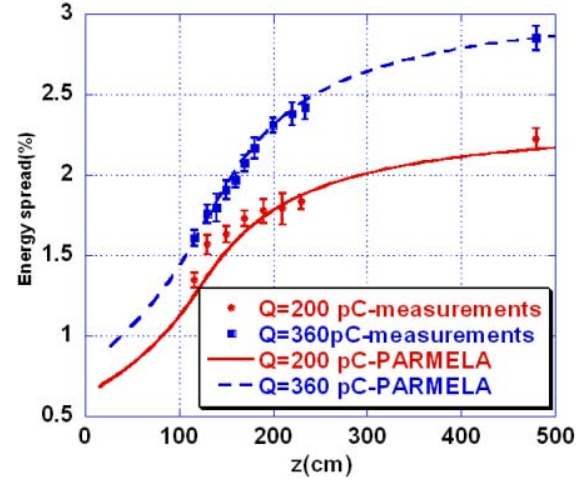

Figure 6: Energy spread vs z evolution.

Table 4: Measured Parameters

\begin{tabular}{|l|l|}
\hline Parameter & Value \\
\hline Energy & $5.5 \mathrm{MeV}$ \\
\hline Spot size, $<\sigma>$ & $450 \mu \mathrm{m}$ \\
\hline Rf phase, $\varphi-\varphi_{\max }$ & $+12^{\circ}$ \\
\hline Pulse shape & Flat top, FWHM=5 psec \\
\hline Solenoid current & $195 \mathrm{~A}$ \\
\hline
\end{tabular}

The excellent agreement between the measurements and the PARMELA model, that does not take into account the wake-fields effect, confirms that the effect of the long emittance-meter bellows is negligible.

\section{HOMDYN Simulation}

In fig.7 we show a comparison between the HOMDYN code and a one day measure at SPARC (measured parameters in table 5). The agreement is quite satisfying if we consider the intrinsic limit of the code. HOMDYN, is indeed a very fast semi-analytical code whose main approximation lies on the assumption that the bunch is a uniformly charged cylinder divided into slices [5].

The difference between the code and the measure is mainly due to the pulse rise/fall time, to the non perfect uniformity of the charge distribution and to the non linearity in the associated electromagnetic fields

neglected by the code. It is interesting to compare the HOMDYN result with the PARMELA fit of the same case as it is shown in fig. 8: the main effect is the disappearance both in the measurements and in PARMELA of the first emittance minimum in the emittance oscillation shown by HOMDYN.
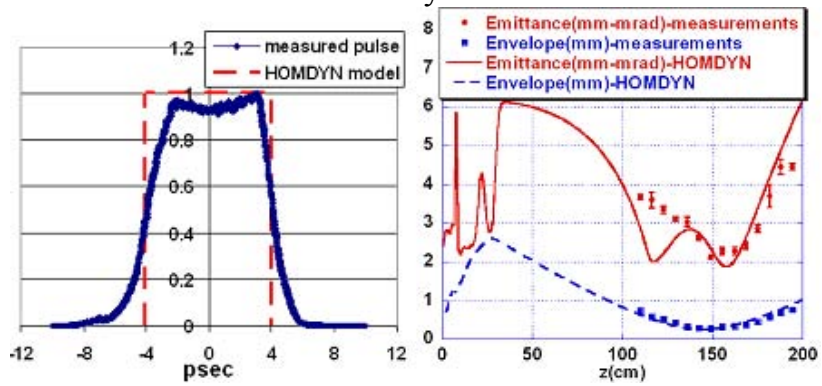

Figure 7: Measured pulse shape compared with the HOMDYN model and corresponding emittance-envelope plot with measurements and HOMDYN results.

Table 5: Measured Parameters

\begin{tabular}{|l|l|}
\hline Parameter & Value \\
\hline Energy & $5.65 \mathrm{MeV}$ \\
\hline Charge & $1 \mathrm{nC}$ \\
\hline Spot size, $<\sigma>$ & $390 \mu \mathrm{m}$ \\
\hline Solenoid current & $209 \mathrm{~A}$ \\
\hline Rf phase, $\varphi$ - $\varphi_{\max }$ & $0^{\circ}$ \\
\hline
\end{tabular}

Figure 8: Emittance plot with HOMDYN-PARMELA vs measurements for the case of fig.7.

\section{REFERENCES}

[1] A. Cianchi et al., "Advanced Measurements at the SPARC Photoinjector", Proceedings of DIPAC07

[2] L. M. Young, PARMELA, Los Alamos National Laboratory report LA-UR-96-1835

[3] L. Giannessi, M. Quattromini, PRST-AB 6120101 (2003), Web site : http://www.tredi.enea.it

[4] M. Ferrario et al., "HOMDYN study for the LCLS RF photoinjector", SLAC-PUB-8400, (2000).

[5] D. Filippetto, "A robust algorithm for beam emittance and trace space evolution reconstruction", SPARC/EBD-07/002, June 2007

[6] M. Ferrario et al., "Experimental results with the SPARC Emittance-meter", these Proceedings

A06 Free Electron Lasers 